\title{
APPLICATION OF L-R METHOD TO SINGLE SERVER FUZZY RETRIAL QUEUE WITH PATIENT CUSTOMERS
}

\author{
J. P. MUKEBA \\ Department of Mathematics and Computer Science \\ High Educational College of Mbuji-Mayi \\ (Institut Supérieur Pedagogique de Mbuji-Mayi) \\ Democratic Republic of Congo \\ e-mail: jpmukeba@yahoo.fr
}

\begin{abstract}
Nowadays, in different domains there are many systems whose processes are described by queueing models. This paper analyzes the characteristics of a queueing model called retrial queue in fuzzy environment using a technique called L-R method. The model considered contains only one server and is frequented only by patient customers. Basing on this technique, customer waiting time and customers number are easily computed in the orbit and in the entire system comparatively to mathematical non-linear programming method. A numerical example is proposed to shows the validity of this approach.
\end{abstract}

\section{Introduction}

Queue model called retrial queue is one of queueing systems which is most used in computer systems, in communication systems, in telephone systems, in supermarkets or at airports when aircrafts are waiting for 2010 Mathematics Subject Classification: 60K20, 03E72.

Keywords and phrases: characteristics of a queueing model, L-R method, mathematical nonlinear programming method, patient customers.

Received July 28, 2016; Revised August 4, 2016

(C) 2016 Scientific Advances Publishers 
landing permission. Generally, analytical analysis of this model is difficult excepted for a few simple models (see [10], [11]). To prevent some inconveniences in its functioning, it is important to know its system characteristics.

In queueing theory literature, retrial queues with patient customers are largely studied in different senses by many researchers, especially in crisp model (e.g., see [1], [11], [2], [8], [15], [6], [9], [21]). Regarding fuzzy model, only a few studies have been devoted to this topic. Precisely, [14] constructed the membership function for a fuzzy retrial queueing system using non-linear programming approach with three fuzzy variables: fuzzified exponential arrival, retrial and service rate. Using fuzzy set theory to deal with a markovian fuzzy retrial queue, [20] computed by alpha-cuts approach and fuzzy arithmetic operations the expected waiting time and the expected number of customers in orbit when the arrival rate, service rate, and retrial rate are triangular fuzzy numbers. In these two last works, the authors have analyzed fuzzy retrial queues characteristics by mathematical non-linear programming method which combines two kinds of fuzzy arithmetics: the one based on Zadeh's extension principle and the one based on alpha-cuts and intervals arithmetic. According to these arithmetics, final results are obtained after a long process which requires successively two steps (e.g., see [16], [13]):

- to transform a fuzzy queue into a family of crisp queues using $\alpha$-cuts approach;

- to develop a set of parametric non linear programs to describe the family of these classical queues by means of system characteristics.

In the present paper, we apply another performance measures computation called L-R method (Left-Right method), recently introduced by [18] to achieve the same task, when some queue parameters are L-R fuzzy numbers. This method is essentially based on L-R fuzzy arithmetic and uses only secant approximations for best results. 
The paper is planned as follows: The second section gives some preliminaries needed in L-R method application. The third section recalls analytical results of a simple retrial queue in crisp model. Based on L-R method description proposed in [18], the fourth section provides a general process to calculate any fuzzy queue characteristic. Following this process, the fifth section solves a numerical example at the end of which all fuzzy retrial queue characteristics are successfully computed in the orbit and in the entire system. The sixth section gives a conclusion.

\section{Preliminaries}

\subsection{Fuzzy set}

Definition 2.1 (Zimmermann [23]). Let $E$ be a classical set or a universe. A fuzzy subset $\widetilde{A}$ (or a fuzzy set $\tilde{A}$ ) in $E$ is defined by the function $\eta_{\tilde{A}}$, called membership function of $\widetilde{A}$, from $E$ to the real unit interval $[0,1]$.

In these conditions, $\eta_{\widetilde{A}}(a)$ is called the grade or the membership degree of $a, \forall a \in \widetilde{A}$. For each $x \in E$ such as $\eta_{\widetilde{A}}(x)=1, x$ is said mean value, modal value or mode of $\tilde{A}$.

Definition 2.2 (Hanss [12]). Let $\widetilde{A}$ be a fuzzy subset in the universe $E$. The alpha-cut $\widetilde{A}_{\alpha}$, the support $\operatorname{supp}(\widetilde{A})$, the height $h g t(\widetilde{A})$, and the core $\operatorname{core}(\tilde{A})$ of $\tilde{A}$, are crisp sets defined, respectively, as follows $\forall a \in[0,1]:$

$$
\begin{gathered}
\widetilde{A}_{\alpha}=\left\{x \in E \mid \eta_{\widetilde{A}}(x) \geq \alpha\right\}, \\
\operatorname{supp}(\tilde{A})=\left\{x \in E \mid \eta_{\widetilde{A}}(x)>0\right\}, \\
\operatorname{hgt}(\tilde{A})=\max \left\{\eta_{\widetilde{A}}(x) \mid x \in E\right\}, \\
\operatorname{core}(\tilde{A})=\left\{x \in E \mid \eta_{\widetilde{A}}(x)=1\right\} .
\end{gathered}
$$


Definition 2.3 (Zadeh [22]). A fuzzy set $\tilde{A}$ is said normal if and only if

$$
\operatorname{hgt}(\tilde{A})=1,
$$

and convex if and only if

$$
\eta_{\widetilde{A}}(\lambda x+(1-\lambda y)) \geq \min \left\{\eta_{\widetilde{A}}(x), \eta_{\widetilde{A}}(y)\right\},
$$

$\forall x, y \in \widetilde{A}, \forall \alpha \in[0,1]$.

\subsection{Fuzzy number}

Definition 2.4 (Buckley and Eslami [4]). A fuzzy number $\widetilde{A}$ is a fuzzy subset of $\mathbb{R}$ such as:

(i) $\operatorname{core}(\widetilde{A}) \neq \emptyset$;

(ii) $\widetilde{A}_{\alpha}$ are all closed and bounded subintervals of $\mathbb{R}$;

(iii) $\operatorname{supp}(\tilde{A})$ is bounded.

Definition 2.5 (Zimmermann [23], p. 59). A fuzzy number $\widetilde{A}$ is said positive if and only if $\forall x<0, \eta_{\widetilde{A}}(x)=0$ and negative if and only if $\forall x>0, \eta_{\widetilde{A}}(x)=0$.

Definition 2.6 (Mukeba et al. [19], p. 71). Let $\widetilde{A}$ and $\widetilde{B}$ be two fuzzy numbers. $\widetilde{A}<\widetilde{B}$ if and only if $\forall x \in \operatorname{supp}(\widetilde{A}), \forall y \in \operatorname{supp}(\widetilde{B}), x<y$.

In the other words,

$$
\widetilde{A}<\widetilde{B} \Leftrightarrow \sup \{\operatorname{supp}(\widetilde{A})\}<\inf \{\operatorname{supp}(\widetilde{B})\} .
$$

\subsection{L-R fuzzy number}

Definition 2.7 (Bouchon [3]). A fuzzy number $\tilde{A}$ is said $L-R$ fuzzy number if only if it exists three real numbers $m, a>0, b>0$ and two positive, continous and decreasing functions $L$ and $R$, from $\mathbb{R}$ to $[0,1]$, such as 


$$
\begin{gathered}
L(0)=R(0)=1, \\
L(1)=0, \quad L(x)>0, \quad \lim _{x \rightarrow \infty} L(x)=0, \\
R(1)=0, \quad R(x)>0, \quad \lim _{x \rightarrow \infty} R(x)=0, \\
\eta_{\tilde{A}}(x)=\left\{\begin{array}{ccc}
L\left(\frac{m-x}{a}\right), & \text { if } & x \in[m-a, m], \\
R\left(\frac{x-m}{b}\right), & \text { if } & x \in[m, m], \\
0, & \text { otherwise. }
\end{array}\right.
\end{gathered}
$$

In these conditions,

- $\widetilde{A}$ is noted $\widetilde{A}=\langle m, a, b\rangle_{L R}$. Sometimes, the notation $\widetilde{A}=\langle m, a, b\rangle_{L R}$ is said the L-R representation of $\tilde{A}$.

$-m$ is the modal value of $\widetilde{A}$.

$-a$ and $b$ are called, respectively, left spread and right spread of $\tilde{A}$.

- According to Equation (2), supp $\tilde{A}$ is the ordinary open interval $\operatorname{supp}(\tilde{A})=] m-a, m+b[$.

- If $L$ and $R$ in Definition 2.7 are given by $L(x)=R(x)=\max (0,1-x)$, the L-R fuzzy number $\widetilde{A}$ is a triangular fuzzy number noted $\widetilde{A}=(a / \mathrm{m} / b)$ or $\widetilde{A}=(a, m, b)$ (see, e.g., [23], [5]). Such an L-R fuzzy number is often used to facilitate the L-R fuzzy numbers illustration. We will use it in Section 5 in the idea to make easy the numerical example proposed.

\subsection{L-R fuzzy numbers arithmetic}

Queues parameters and queues systems characteristics being often positive numbers, we are intersested in this subsection only by arithmetic operations on positive L-R fuzzy numbers. Regarding this arithmetic, some results exist and are well known in the literature. For two L-R fuzzy numbers $\tilde{M}=\langle m, a, b\rangle_{L R}$ and $\tilde{N}=\langle n, c, d\rangle_{L R}$, the sum and the 
difference are also L-R fuzzy numbers (see [7]), but the product and the quotient do not be L-R fuzzy numbers (see [12]). For multiplication and division, L-R method needs only their secant approximations formulas for best results (see [17]). Thus, all calculations of fuzzy queues characteristics pass by the following formulas (see [18], p. 60):

$$
\begin{gathered}
\tilde{M}+\tilde{N}=\langle m+n, a+c, b+d\rangle_{L R}, \\
\tilde{M}-\tilde{N}=\langle m-n, a+d, b+c\rangle_{L R}, \\
\tilde{M} \cdot \tilde{N} \approx\langle m \cdot n, m c+n a-a c, m d+n b+b d\rangle_{L R}, \\
\frac{\tilde{M}}{\tilde{N}} \approx\left\langle\frac{m}{n}, \frac{m d}{n(n+d)}+\frac{a}{n}-\frac{a d}{n(n+d)}, \frac{m c}{n(n-c)}+\frac{b}{n}+\frac{b c}{n(n-c)}\right\rangle_{L R} .
\end{gathered}
$$

\section{The Crisp Model of Retrial Queue}

\subsection{Description of $M / M / 1$ retrial queue}

The basic model of a simple markovian retrial queue, briefly noted "M/M/ $1 / 1-\mathrm{R}$ " is given in ([2], p. 300). It is represented in Figure 1 below. Let us assume as in ([11], p. 159) that a single class of customers from calling source arrives to the system according to a Poisson flow with rate $\lambda$.

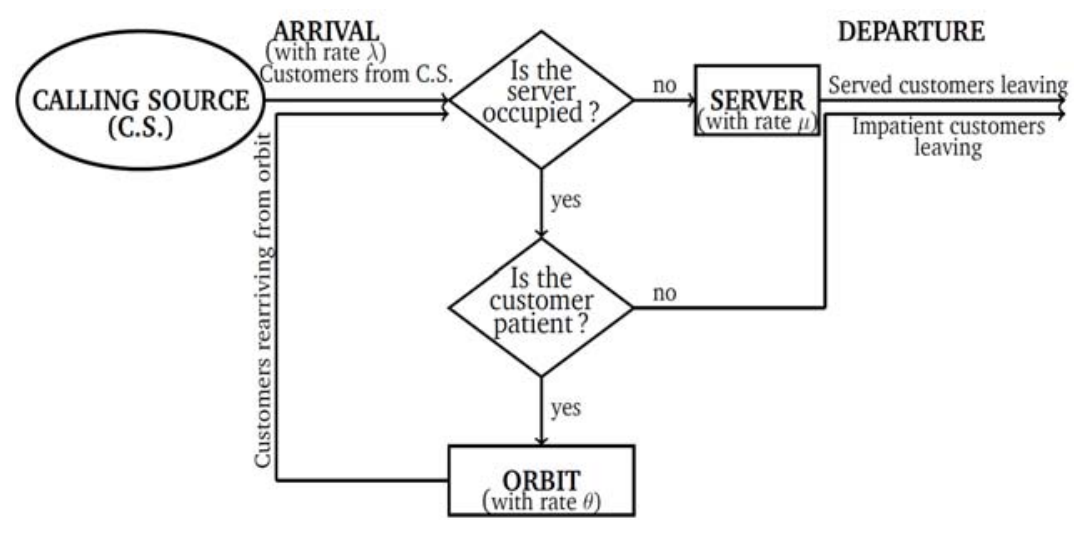

Figure 1. Schematic diagram of a simple traditional retrial queue. 
(i) If a primary customer finds the server available, he gets in, receives a service and leaves the system. It is assumed that the server offers an exponential service with rate $\mu$.

(ii) If he finds the server busy, he may do two choices:

(a) To leave the system altogether (impatience case);

(b) To return later to the server (patience case). While away, the customer is said to be in orbit.

(iii) From the orbit, he can only check the server status by rearriving at the server. Such an event is called a retrial. It is assumed that all inter-retrials are exponentially distributed with parameter $\theta$.

(iv) The customer located in the orbit repeats the request after some random time until the server is ready to receive him, otherwise he abandons the system.

(v) In this work, we will assume that:

(a) No customer leaves the system due to impatience, this implies that the total output flow equals to $\mu$;

(b) The orbit capacity is assumed to be infinite;

(c) All processes in the system are i.i.d. (independent and identically distributed).

In these conditions, the orbit is like a queue in witch customers spend time waiting to be served. In orbit, a customer cannot monitor the status of the server. In particular, once the server becomes free, there is a delay in time until a customer in orbit realizes that the server is free and begins service. Also, it is excluded that customers queue in orbit. From the orbit, the order of service depends on the random order in which customers return to check the system status and the random chance that a server is available at the moment when the customer returns to it. In particular, customers do not generally receive service by first-in first-out discipline. 


\subsection{Characteristics of $M / M / 1$ retrial queue}

Let us denote by $W_{0}, L_{0}, W_{s}, L_{s}, W$, and $L$, respectively, the mean time spent in orbit, the expected number of customers in orbit, the customer waiting time in the server, the expected number of customers in the server, the average waiting time in the system, and the expected number of customers in the system. Since, there is only one server, then $L_{s}=\frac{\lambda}{\mu}$ and $W_{s}=\frac{1}{\mu}$.

Moreover,

$$
W=W_{0}+W_{s}
$$

and

$$
L=L_{0}+L_{s}
$$

With the above assumptions, Bolch et al. ([2], pp. 301-302) and Gross et al. ([11], p. 162), show that if the queue is stable, that is $\lambda<\mu$, then the characteristics $L_{0}, L, W_{0}$, and $W$ are computed by the following crisp formulas at steady state:

$$
\begin{gathered}
W_{0}=\frac{\lambda(\mu+\theta)}{\mu \theta(\mu-\lambda)}, \\
L_{0}=W_{0} \cdot \lambda=\frac{\lambda^{2}(\mu+\theta)}{\mu \theta(\mu-\lambda)}, \\
W=\frac{\lambda+\theta}{\theta(\mu-\lambda)}, \\
L=W \cdot \lambda=\frac{\lambda(\lambda+\theta)}{\theta(\mu-\lambda)} .
\end{gathered}
$$




\section{The Fuzzy Model}

\subsection{Characteristics of fuzzy retrial queue}

Let us reconsider all assumptions of a retrial queue in Section 3, and assume that all rates $\lambda, \mu$, and $\theta$ are L-R fuzzy numbers noted $\tilde{\lambda}, \tilde{\mu}$, and $\widetilde{\theta}$. In these conditions, the model becomes the fuzzy model noted "FM/FM/1 / 1- R", where FM denotes a fuzzified exponential distribution. Further to this change in queue parameters nature, the crisp performance measures formulas in Equations (16), (17), (18), and (19) change and become, respectively:

$$
\begin{gathered}
\widetilde{W}_{0}=\frac{\tilde{\lambda}(\tilde{\mu}+\tilde{\theta})}{\tilde{\theta} \tilde{\mu}(\tilde{\mu}-\tilde{\lambda})}, \\
\widetilde{L}_{0}=\tilde{W}_{0} \cdot \tilde{\lambda}, \\
\widetilde{W}=\frac{\tilde{\lambda}+\tilde{\theta}}{\tilde{\theta}(\tilde{\mu}-\tilde{\lambda})}, \\
\tilde{L}=\tilde{W} \cdot \tilde{\lambda} .
\end{gathered}
$$

Here, we do not use the classical arithmetic to compute system characteristics. $\tilde{\lambda}, \tilde{\mu}$, and $\tilde{\theta}$ being fuzzy numbers, we must use the fuzzy arithmetic. Instead of mathematical non-linear programming method used in the two aforesaid works, we use now L-R method whose the process is proposed in the following subsection to compute these characteristics. But before to launch computation, it is required to be sure that the queue stability condition is verified. By analogy to crisp model and according to Equation (5), such a fuzzy queue is stable if only if the input flow $\tilde{\lambda}$ is less than the output flow $\tilde{\mu}$, in the other words:

$$
\sup \{\operatorname{supp}(\tilde{\lambda})\}<\inf \{\operatorname{supp}(\tilde{\mu})\} \text {. }
$$




\subsection{L-R method process}

As indicated above, L-R method consists in using L-R fuzzy arithmetic rectricted to secant approximations to compute fuzzy queues characteristics. That is mean, using L-R method, arithmetic operations in Equations (20), (21), (22), and (23) are computed by corresponding formula chosen among Equations (10), (11), (12), and (13) after putting all queue parameters in their L-R representations. Suppose, for example, that we are in want to determine a fuzzy queue characteristic whose the crisp formula is given by

$$
K=f\left(x_{1}, x_{2}, \ldots, x_{n}\right),
$$

where $f$ is a real multi-valued function and $x_{1}, x_{2}, \ldots, x_{n}$ the crisp queue parameters. The fuzzy formula of this characteristic is

$$
\widetilde{K}=\tilde{f}\left(\tilde{x}_{1}, \tilde{x}_{2}, \ldots, \tilde{x}_{n}\right),
$$

where $\tilde{f}$ is a fuzzy multi-valued function and $\tilde{x}_{1}, \widetilde{x}_{2}, \ldots, \widetilde{x}_{n}$ the fuzzy queue parameters. Basing on L-R method described in ([18], p. 60), $\widetilde{K}$ can be determined by the following process:

(1) Determine L-R representations of fuzzy numbers $\tilde{x}_{1}, \tilde{x}_{2}, \ldots, \tilde{x}_{n}$ and substitute them in Equation (25). If $\tilde{x}_{i}=\left\langle m_{i}, \varphi_{i}, \omega_{i}\right\rangle_{L R}$ for $1 \leq i \leq n$, Equation (25) becomes:

$$
\widetilde{K}=\widetilde{f}\left(\left\langle m_{1}, \varphi_{1}, \omega_{1}\right\rangle_{L R},\left\langle m_{2}, \varphi_{2}, \omega_{2}\right\rangle_{L R}, \ldots,\left\langle m_{n}, \varphi_{n}, \omega_{n}\right\rangle_{L R}\right) .
$$

(2) Compute the expression obtained in Equation (26) applying L-R fuzzy arithmetic rectricted to secant approximations. For these operations, only formula in Equations (10), (11), (12), and (13) are used.

(3) The final result in the second step is an L-R fuzzy number $\widetilde{K}$ such as

$$
\widetilde{K}=\langle m, \varphi, \omega\rangle_{L R},
$$

where $m, \varphi, \omega$, and $] m-\varphi, m+\omega[$ represent, respectively, the modal value, the left spread, the right spread, and the support of $\widetilde{K}$. 
(4) So, the performance measure computed is a fuzzy number whose modal value and support are, respectively, $m$ and $] m-\varphi, m+\omega[$. In the other words, $\widetilde{K}$ is an imprecise real number whose value is approximately between $m-\varphi$ and $m+\omega$ and whose the most possible value is $m$.

Remark 4.1. Different queue rates being often strictly positive numbers, we will exclude in computation all cases of fuzzy numbers $\tilde{X}$ such as $0 \in \operatorname{supp}(\tilde{X})$. This exception eliminates absurd results due to the presence of zero as a denominator. Moreover, the queue being stable, we do not obtain absurd or negative results due to the presence of the factor $\tilde{\mu}-\tilde{\lambda}$ in denominators of Equations (20), (21), (22), and (23).

The following section shows the practical use of this approach through an example treated in [20].

\section{Numerical Example}

\subsection{Problem}

In a packet switching network, we considered a computer network in which there are a group of host computers, connected to interface message processors. Message arrives at the host computer following a Poisson stream. If the host computer wishes to transmit the message to another host computer, it must send the message and the final address to the interface message processor to which it is associated. If the processor is free the message is accepted. Otherwise, the message comes back to the host computer and is stored in a buffer to be transmitted some time later. The buffer in the host computer, the interface processor and the transmission policy correspond to the orbit, the server and the retrial discipline, respectively, in the queuing terminology. Concerned with system efficiency, the management wants to obtain the system characteristics, including the expected waiting time and the number of 
customer in the orbit. The arrival of message to the system is about 16 per hour. From buffer, the message recalls the processor around 8 times per hour. The processor treats about 21 message per hour.

\subsection{Solution}

Let us assume as in [20] that arrival rate, retrial rate and service rate in the system are triangular fuzzy numbers represented, respectively, by $\tilde{\lambda}=(15,16,18), \tilde{\mu}=(19,21,22)$, and $\tilde{\theta}=(1,8,22)$. Theseparameters being L-R fuzzy numbers, we can use the process described in Subsection 4.2 to analyze queue characteristics enumerated in equations from (20) to (23).

(1) Putting fuzzy parameters $\tilde{\lambda}, \tilde{\theta}$, and $\tilde{\mu}$ in their L-R representations, we get

$$
\begin{aligned}
& \tilde{\lambda}=\langle 16,1,2\rangle_{L R}, \\
& \tilde{\theta}=\langle 8,7,14\rangle_{L R}, \\
& \tilde{\mu}=\langle 21,2,1\rangle_{L R} .
\end{aligned}
$$

(2) Substituting these L-R representations, respectively, in Equations (20), (21), (22), and (23), we obtain

$$
\begin{gathered}
\tilde{W}_{0}=\frac{\langle 16,1,2\rangle_{L R} \cdot\left[\langle 21,2,1\rangle_{L R}+\langle 8,7,14\rangle_{L R}\right]}{\left[\langle 8,7,14\rangle_{L R} \cdot\langle 21,2,1\rangle_{L R}\right] \cdot\left[\langle 21,2,1\rangle_{L R}-\langle 16,1,2\rangle_{L R}\right]}, \\
\tilde{L}_{0}=\langle 16,1,2\rangle_{L R} \cdot \tilde{W}_{0}, \\
\tilde{W}=\frac{\langle 16,1,2\rangle_{L R}+\langle 8,7,14\rangle_{L R}}{\langle 8,7,14\rangle_{L R} \cdot\left[\langle 21,2,1\rangle_{L R}-\langle 16,1,2\rangle_{L R}\right]}, \\
\tilde{L}=\langle 16,1,2\rangle_{L R} \cdot \tilde{W} .
\end{gathered}
$$


(3) Computing characteristics in Equations (31), (32), (33), and (34) using formula among (10), (11), (12), and (13), we obtain $\widetilde{L}_{0}, \widetilde{W}_{0}, \widetilde{L}$, and $\widetilde{W}$ as follows:

$$
\begin{aligned}
& \tilde{W}_{0}=\frac{\langle 16,1,2\rangle_{L R} \cdot\left[\langle 21,2,1\rangle_{L R}+\langle 8,7,14\rangle_{L R}\right]}{\left[\langle 8,7,14\rangle_{L R} \cdot\langle 21,2,1\rangle_{L R}\right] \cdot\left[\langle 21,2,1\rangle_{L R}-\langle 16,1,2\rangle_{L R}\right]} \\
& =\frac{\langle 16,1,2\rangle_{L R} \cdot\langle 29,9,15\rangle_{L R}}{\langle 168,16+147-14,8+294+14\rangle_{L R} \cdot\langle 5,4,2\rangle_{L R}} \\
& =\frac{\langle 464,144+29-9,240+58+30\rangle_{L R}}{\langle 840,672+745-596,336+1580+632\rangle_{L R}} \\
& =\frac{\langle 464,164,328\rangle_{L R}}{\langle 840,821,2548\rangle_{L R}} \\
& =\langle 0.552,0.464,41.131\rangle_{L R} ; \\
& \widetilde{L}_{0}=\langle 16,1,2\rangle_{L R} \cdot \tilde{W}_{0} \\
& =\langle 16,1,2\rangle_{L R} \cdot\langle 0.552,0.464,41.131\rangle_{L R} \\
& =\langle 8.832,7.424+0.552-0.464,658.096+1.104+82.262\rangle_{L R} \\
& =\langle 8.832,7.512,741.462\rangle_{L R} \\
& \tilde{W}=\frac{\langle 16,1,2\rangle_{L R}+\langle 8,7,14\rangle_{L R}}{\langle 8,7,14\rangle_{L R} \cdot\left[\langle 21,2,1\rangle_{L R}-\langle 16,1,2\rangle_{L R}\right]} \\
& =\frac{\langle 24,8,16\rangle_{L R}}{\langle 8,7,14\rangle_{L R} \cdot\langle 5,4,2\rangle_{L R}} \\
& =\frac{\langle 24,8,16\rangle_{L R}}{\langle 40,39,114\rangle_{L R}} \\
& =\left\langle\frac{3}{5}, \frac{382}{770}, \frac{1576}{40}\right\rangle_{L R} \\
& =\langle 0.6,0.4961,39.4\rangle_{L R} ;
\end{aligned}
$$




$$
\begin{aligned}
\widetilde{L} & =\langle 16,1,2\rangle_{L R} \cdot \widetilde{W} \\
& =\langle 16,1,2\rangle_{L R} \cdot\left\langle\frac{3}{5}, \frac{382}{770}, \frac{1576}{40}\right\rangle_{L R} \\
& =\left\langle\frac{48}{5}, \frac{3096}{385}, \frac{3552}{5}\right\rangle_{L R} \\
& =\langle 9.6,8.0415,710.4\rangle_{L R} .
\end{aligned}
$$

(4) According to Definition 2.7, the modal values of $\widetilde{W}_{0}, \widetilde{L}_{0}, \widetilde{W}, \widetilde{L}$ are, respectively, $m_{\widetilde{W}_{0}}=0.552, m_{\widetilde{L}_{0}}=8.83, m_{\widetilde{W}}=0.6, m_{\widetilde{L}}=9.6$ and their supports are the following open intervals:

$$
\begin{aligned}
& \left.\operatorname{supp}\left(\widetilde{W}_{0}\right)=\right] 0.552-0.464,0.552+41.131[=] 0.088,41.6[, \\
& \left.\operatorname{supp}\left(\widetilde{L}_{0}\right)=\right] 8.83-7.51,8.83+741.46[=] 1.32,750[ \\
& \operatorname{supp}(\widetilde{W})=] 0.6-0.4961,0.6+39.4[=] 0.103,40[ \\
& \operatorname{supp}(\widetilde{L})=] 9.6-8.0415,9.6+710.4[=] 1.558,720[
\end{aligned}
$$

\subsection{Results}

- According to item 4 of Subsection 4.2, the modal value $m_{\widetilde{W}_{0}}=0.552$ and the support $\left.\operatorname{supp}\left(\widetilde{W}_{0}\right)=\right] 0.088,41.6[$ indicate that the message waiting time $\widetilde{W}_{0}$ in the buffer is approximately between 0.088 and 41.6 hours. Its most possible value is 0.552 hour or 33 minutes. Likewise,

- The messages number $\widetilde{L}_{0}$ in the buffer is between 1 and 750. Its most possible value is 8 .

- The message waiting time $\widetilde{W}$ in the system is approximately between 0.103 and 40 hours. Its most possible value is 0.6 hour or 36 minutes.

- The messages number $\tilde{L}$ in the system is between 1 and 720 . Its most possible value is 9 . 


\section{Conclusion}

In this paper, L-R method is introduced in fuzzy queueing theory to evaluate characteristics of a fuzzy retrial queue with patient customers when queue parameters are L-R fuzzy numbers. To get the results, this method does not solve any program from mathematical non-linear programming method. This fact constitutes a major advantage which make it short and flexible compared to this one. Attracted by this flexibility, we wish to know if there is one possibility to extend it to fuzzy queues models whose parameters are not L-R fuzzy numbers. This question remains operational for future research. With its help, messages number and message waiting time are successfully computed in the buffer and in the entire system in Section 5. Moreover, it is wellestablished in fuzzy logic literature that for system designers and practionners, analytical results obtained under fuzzy environment are more useful than those usually obtained under conventional model, because they are very realistic and very informative.

\section{References}

[1] J. R. Artalejo and A. Gomez-Corral, Retrial Queueing Systems, A Computational Approach, Springer-Verlag, Berlin Heidelberg, 2008.

[2] G. Bolch, S. Greiner, H. De-Meer and K. S. Trivedi, Queueing Networks and Markov Chains, 2nd Edition, John Wiley \& Sons, New Jersey, 2006.

[3] B. Bouchon-Meunier, La Logique Floue et Ses Applications, Addison-Wesley France, Paris, 1995.

[4] J. J. Buckley and E. Eslami, An Introduction to Fuzzy Logic and Fuzzy Sets, Springer-Verlag, Berlin Heidelberg, 2002.

[5] J. J. Buckley, E. Eslami and T. Feuring, Fuzzy Mathematics in Economics and Engineering, Physica-Verlag, Heidelberg, 2010.

[6] J. E. Diamond and A. S. Alpha, Approximation method for M/PH/I retrial queues with phase type inter-retrial times, Eur. Journ. Oper. Res., 113 (1999), 620-6312.

[7] D. Dubois and H. Prade, Fuzzy Sets and Systems: Theory and Applications, Academic Press, New York, 1980. 
[8] A. N. Dudin, A. Krishnamoorthy, A. V. C. Joshua and G. V. Tsarenkov, Analysis of the BMAP/G/I retrial system with search of customers from the orbit, Eur. J. Oper. Res., 157 (2004), 169-179.

[9] G. I. Falin and J. G. C. Templeton, Retrials Queues, Springer-Sciences + Business Media, London, 1997.

[10] I. Florea and C.-S. Nanau, An algorithmic approach of retrial queueing system with one serving station, Part I: The description of the simulation algorithm, Bulletin of the Transilvania University of Brasov; Série III: Mathematics, Informatics, Physics 6(2) (2013), 95-106.

[11] D. Gross, J. F. Shortie, J. M. Thompson and C. M. Harris, Fundamentals of Queueing Theory, Fourth Edition, Wiley, New Jersey, USA, 2008.

[12] M. Hanss, Applied Fuzzy Arithmetic, An Introduction with Engineering Applications, Springer-Verlag, Berlin Heidelberg, 2005.

[13] M. Jeeva and E. Rathnakumari, Fuzzy cost computations of M/M/1 and M/G/1 queueing models, British Journal of Mathematics and Computer Science 4(1) (2014), 120-132.

[14] J.-C. Ke, H.-I. Huang and C.-H. Lin, On retrial queueing model with fuzzy parameter, Physica A 374 (2007), 272-280.

[15] M. J. Lopez-Herrero, On the number of customers served in the M/G/I retrial queue: First moments and maximum entropy approach, Comput. Oper. Res. 29 (2002), 1739-1757.

[16] K. J. R. Mary, P. Monica and S. Mythili, Performance measures of FM/FM/1 queueing system with N-policy, International Journal of Physics and Mathematical Sciences 4(3) (2014), 5-10.

[17] J. P. Mukeba, R. Mabela and B. Ulungu, Sur la multiplication des nombres flous de type L-R, Annals of Faculty of Sciences, University of Kinshasa, 1 (2014), 33-40.

[18] J. P. Mukeba, R. Mabela and B. Ulungu, Computing fuzzy queueing performance measures by L-R method, Journal of Fuzzy Sets Valued Analysis 1 (2015), 57-67.

http://dx.doi.org/10.5899/2015/jfsva-00226

[19] J. P. Mukeba, R. Mabela and B. Ulungu, Performance measures of a product form queueing network with fuzzy parameters, Journal of Fuzzy Sets Valued Analysis 1 (2015), 68-77.

http://dx.doi.org/10.5899/2015/jfsva-00228

[20] W. Rita and L. Robert, Application of fuzzy set theory to retrial queues, International Journal of Algorithms, Computing and Mathematics 2(4) (2009), 9-18.

[21] T. Yang and J. G. C. Templeton, A survey on retrial queues, Queueing Systems 2 (1987), 201-203. 
[22] L. A. Zadeh, Fuzzy sets, Information and Control 8 (1965), 338-353.

http://dx.doi.org/10.1016/S0019-9958(65)90241-X

[23] H.-J. Zimmermann, Fuzzy Set Theory and its Applications, Fourth Edition, Springer Science + Business Media, New-York, 2001.

http://dx.doi.org/10.1007/978-94-010-0646-0 